\title{
Novel Linear Ion Trap Mass Analyzer Composed of Four Planar Electrodes
}

\author{
Yishu Song, Guangxiang Wu, Qingyu Song, R. Graham Cooks, \\ and Zheng Ouyang \\ Department of Chemistry, Purdue University, West Lafayette, Indiana, USA
}

Wolfgang R. Plass

II Physikalisches Institut, Justus-Liebig-Universität Giessen, Giessen, Germany

\begin{abstract}
A novel linear ion trap mass analyzer was developed using just four elongated planar electrodes, mounted in parallel, and employing an RF potential for ion trapping in the radial and axial directions. Mass analysis was achieved using the mass-selective instability scan with ion ejection in the radial direction. The performance of this new device was characterized in comparison with the 6-electrode rectilinear ion trap (RIT) from which it is derived. The 4-electrode trap gives optimum performance in an asymmetric geometry, just like the original optimized 6-electrode RIT. The strong RF fringing fields at the ends of the RF rods account for axial ion trapping without use of extra electrodes or an axial DC voltage. Field calculations and simulations have been carried out to study the trapping potential inside RITs with various configurations. Demonstrated capabilities include analysis of externally injected ions with mass resolution in excess of 1000 and a mass/charge range of 650 Th as well as tandem mass spectrometry capabilities. The geometric simplicity and performance characteristics of the 4-electrode RIT make it particularly attractive in the development of next generation miniaturized mass spectrometers. (J Am Soc Mass Spectrom 2006, 17, 631-639) (c) 2006 American Society for Mass Spectrometry
\end{abstract}

I on trap mass spectrometry [1] is playing an increasingly important role in modern instrumental analysis. Capabilities for identifying and quantifying high and low molecular weight compounds, both in pure form and as components of complex mixtures, and with high sensitivity and specificity, facilitate the investigation of chemical or biochemical systems. The attractiveness of ion trap mass spectrometry is enhanced by the fact that high-quality analytical performance is achieved using a relatively simple device. In particular, the ability to perform multi-stage tandem mass spectrometry using a single analyzer in a single instrument represents a major advantage.

Electrodynamic ion traps date back to the pioneering work of Wolfgang Paul et al. in the 1950s [2]. These authors first described the three-dimensional electric quadrupole field established by three electrodes with hyperbolic surfaces and their ion trapping capabilities. When used as an ion trap, the ring electrode is supplied with a fixed megahertz radio frequency (RF) voltage and the two endcap electrodes are normally grounded. The invention by Stafford [3] of the mass-selective instability scan, which is achieved by scanning of the RF

Published online February 28, 2006

Address reprint requests to Professor R. Graham Cooks, and Dr. Zheng Ouyang, Department of Chemistry, Purdue University, 560 Oval Dr., West Lafayette, IN 47907, USA. E-mail: cooks@purdue.edu, ouyang@purdue.edu. amplitude, allowed the Paul trap to be used in a straightforward way as a mass analyzer. Different ion trap geometries have evolved as modifications to the original Paul design, either for performance improvement or as adaptations for specific applications. The manipulation to the higher-order fields of the trap by stretching its geometry [4] or changing the electrode shapes [5] has been used to eliminate small mass shifts and so to improve mass resolution. While the majority of commercial ion trap mass spectrometers employ the Paul geometry, difficulty in the accurate implementation of hyperbolic electrode structures in smaller traps more suited for portable mass spectrometers, as well as the relaxed analytical performance criteria for applications of portable analytical instruments, has led to intensive explorations of geometrically simpler alternatives. Accordingly, the cylindrical ion trap (CIT) [6] has been developed into a mass analyzer by empirical optimization of its geometry, [7] one in which a cylindrical electrode and planar endcaps replace the hyperbolic ring electrode and hyperbolic endcap electrodes of the conventional Paul trap. A mass/charge range up to $600 \mathrm{Th}$ with unit resolution together with capabilities for recording product ion tandem mass spectra can be obtained using this significantly simplified geometry, which is easily fabricated and miniaturized to the sub-mm [8] and even into the micron [9] size range. 
Both conventional Paul traps and CITs have inherently limited ion trapping capacity, due to the 3D nature of the RF trapping field which confines trapped ions to a point at the center of the device [10, 11]. Provided that space charge effects are held constant, analytical performance of ion traps increases with the number of trapped ions, which tend to be accumulated at or near this central point. These difficulties lead to increased interest in linear traps in which ions are trapped along a line, rather than at a point. So severe are the limitations of the Paul type traps that the actual number of ions trapped in an instrument of conventional size (few $\mathrm{mm}$ to $1 \mathrm{~cm}$ internal radius) is limited to only a few hundred under conditions of good resolution [12]. Further effort at optimizing higher-order fields inside 3D traps to maintain mass resolution while increasing the number of trapped ions has led to ingenious solutions [10], although these have as yet met with only limited success.

In addition to the limitation in the total number of ions that can be trapped in a Paul 3D trap, these devices have a low trapping efficiency for externally injected ions due to the RF field alternating against the ions injected through the endcap electrode hole. Linear ion traps $[13,14]$ improve both the trapping capacity and trapping efficiency for externally injected ions. To circumvent the mechanical difficulties analogous to those which hindered miniaturization of the Paul trap, a modified form of linear ion traps, the rectilinear ion trap (RIT), has been developed [15]. This mass analyzer consists of two pairs ( $x$ and $y$ ) of planar electrodes mounted in parallel as the counterparts of the hyperbolic rod set, and a pair of z-electrodes that are used as the endcaps. Like the CIT, the RIT is a mass analyzer of simplified geometry, but it is the simplified analog of the higher performance LIT, while the CIT is the geometrically simplified analog of the 3D Paul trap. Significantly better performance has been achieved using RITs compared to CITs of similar dimension operated under similar conditions. As expected, many of the advantages of the RIT are the result of its increased trapping capacity and improved injection efficiency [15-18].

The structure of linear ion traps is derived from the quadrupole mass filter with a pseudopotential well in the $x-y$ plane (perpendicular to the ion optical axis) generated by an RF field. Instead of having a pseudopotential well in the third dimension as is the case in a 3D trap, linear ion traps have an additional DC potential well in the $\mathrm{z}$ direction formed by the DC voltages applied between the end sections and the RF electrodes [19]. The end sections can be simply two planar lens elements [14, 15] or two additional sections of RF electrodes [13]. Unlike mass analysis using a 3D trap with fixed ratios of the dimensions in all three directions, mass analysis in a linear trap is not inherently dependent on the $\mathrm{z}$ dimension and $\mathrm{a} \mathrm{z}$ dimension much greater than the $\mathrm{x}$ and $\mathrm{y}$ dimensions is used to establish a cylindrical trapping volume that is considerably larger than the spherical volume generated by a 3D ion trap. This results in a significantly increased trapping capacity fundamentally associated with trapping along a line versus at a point $[1,13,14,19]$. In addition, when dual-phase RF is used, the ions can be injected into the linear trap along the axial direction and thus not subject to a direct RF retarding and accelerating field which leads to the increased trapping efficiency for external ion injection. These advantages are shared by both the higher quality field versions of linear ion traps and by the simplified RIT format.

The use of an RF-generated, rather than a DCgenerated, trapping potential well is advantageous when linear ion traps are used for certain applications including ion/ion reactions [20,21], electron caption dissociation [22], and electron-transfer dissociation [23, 24], where particles with opposite charges need to be trapped simultaneously. This requirement has been met for RF-only traps by superimposing a pseudopotential well along the $z$ direction by applying AC signals on the end lenses [20,24] or using an unbalanced RF [21] for the linear ion trap, although this requires additional electronic controls.

The effects of $\mathrm{z}$ direction DC potentials on ion trapping in the original 6-electrode RIT were further studied and the results suggested that the axial DC potential is unnecessary for ion trapping and subsequent mass analysis. That is, the 6-electrode RIT structure can be replaced by a 4-electrode structure which is asymmetrical in the x-y plane (the "stretched" geometry). This additional simplification to the RIT geometry in which the z-electrodes are removed is described here; the resulting device is composed of just four planar RF electrodes. This highly simplified device, which we term the 4-electrode RIT, employs a pure RF potential for ion trapping in both the radial and axial directions and functions as a linear ion trap without any performance loss compared to the original 6-electrode RIT. The geometric simplicity and the convenience of compensating for the trapping capacity loss due to the shrinking of the radial dimension by increasing its length, makes this device particularly significant for the development of the next generation of miniaturized mass spectrometers and for future instruments which will employ arrays of RITs arranged in two- and three-dimensions.

\section{Experimental}

A previously characterized RIT [15] has been used for comparative purposes in this study. Each $\mathrm{x}$ - or $\mathrm{y}$ electrode is $40.0 \mathrm{~mm}$ long. The half-distance between the $\mathrm{x}$-electrode pair $\left(\mathrm{x}_{0}\right)$ is $5.0 \mathrm{~mm}$ and the distance between the $y$-electrode pair $\left(\mathrm{y}_{0}\right)$ is adjustable. The closest gap between adjacent electrodes is fixed at 1.6 $\mathrm{mm}$. Centrally located on each x-electrode is a slit 15.0 $\mathrm{mm}$ long and $1.0 \mathrm{~mm}$ wide. The 4-electrode RIT was obtained by removing the z-electrodes from the original RIT assembly and stretching the geometry in the $x$ 
direction by using a shorter distance, $3.8 \mathrm{~mm}$, between the y-electrode pair $\left(\mathrm{y}_{0}\right)$.

Both the 6- and 4-electrode RITs were tested using a modified prototype ThermoFinnigan ITMS [25]. An RF signal $(1.1 \mathrm{MHz})$ was applied on the y-electrodes while the $x$-electrodes were virtually grounded so as to form an RF trapping field in the $x-y$ plane. Internal electron impact (EI) ionization was used to ionize the vapors of molecules leaked into the trap, using a heated filament to provide electrons that were injected into the trap radially through the slit in one of the x-electrodes, unless stated otherwise. For experiments using external ion injection, a GCQ EI/CI source was modified and mounted in the manifold. Ions generated by $70 \mathrm{eV}$ electron impact were extracted from the source and injected (gated) into the trap on the z-axis in pulses of selected intervals and duration. The filament and ion injection controls were modified from the ITMS filament and electron gate control.

The RIT assembly was installed into its vacuum manifold some $78.0 \mathrm{~mm}$ distant from the manifold walls, as shown in Figure 1b. A DeTech 397 detector assembly (Detector Technology, Inc., Palmer, MA) was used in the experiment. It has a stainless steel case that shields the conversion dynode and the electron multiplier, and helps to minimize interference from the applied high voltages. An opening of $12.5 \mathrm{~mm}$ diameter on the detector casing allows ions to enter the detector. Electric connections and wires were carefully placed to minimize possible fringing fields along the $\mathrm{z}$ axis of the RIT.

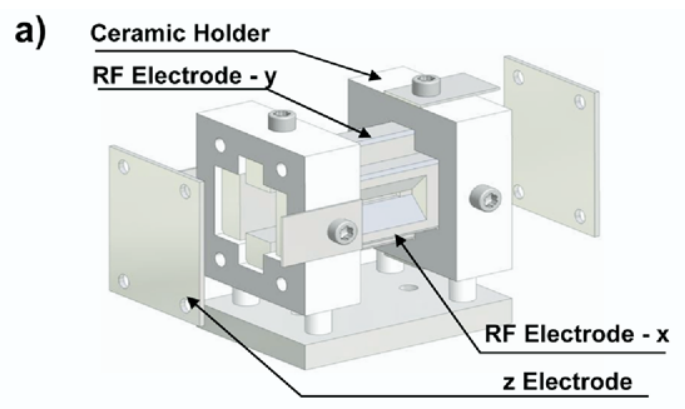

b)

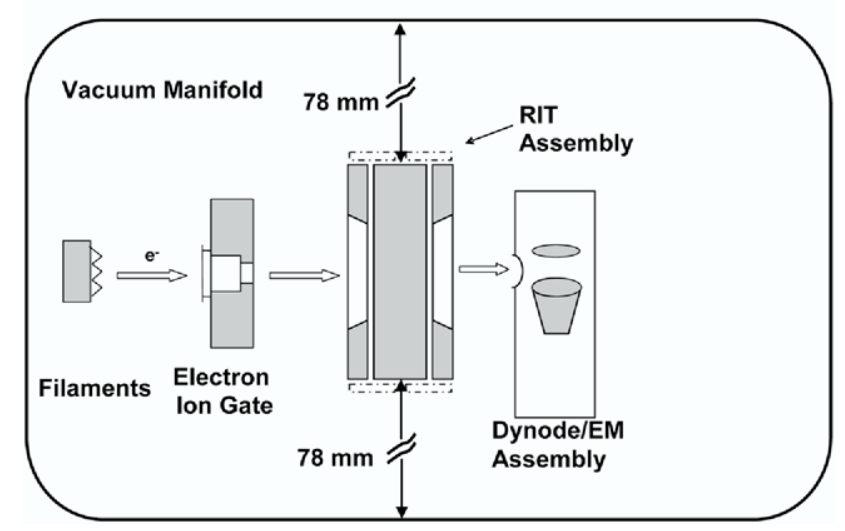

Figure 1. RIT assembly (a) and the instrument setup for internal EI experiments (b).
Trapped ions were mass-selectively ejected by scanning the RF amplitude at a rate of $16,665 \mathrm{Th} / \mathrm{s}$. A supplementary low voltage AC signal, generated using a WaveTek 395 arbitrary waveform generator (WaveTek, San Diego, CA) and amplified by a Balun amplifier, was applied between the x-electrodes to provide a dipolar field for resonance ejection to facilitate ion ejection during the RF scan. This field was also used for ion excitation in the collision-induced dissociation (CID) experiments. Either RF/DC or stored waveform inverse Fourier transform (SWIFT) [26] waveform isolation was used for ion isolation in $\mathrm{MS}^{\mathrm{n}}$ experiments, as indicated. The SWIFT waveforms were calculated using the Ion Trap Simulation program (ITSIM) 5.0 research version [27], generated using WaveForm DSP2 software (version 2.02), and then deployed with the Wavetek 395-64k $100 \mathrm{MHz}$ Synthesized Arbitrary Waveform Generator. Ions ejected from the RIT were detected using the DeTech detector assembly noted above, which was equipped with an electron multiplier, operated at $-1200 \mathrm{~V}$ and with a conversion dynode operated at $-5000 \mathrm{~V}$. The signal was first amplified using the preamplifier in the ITMS and then acquired using a digital oscilloscope (model TDS 540; Tektronix Beaverton, OR) at a sampling rate of $250 \mathrm{~K}$ samples/s.

Helium was used as buffer gas at an indicated pressure of ca. $8.5 \times 10^{-5}$ torr, measured using a Bayert-Alpert type ionization gauge. Headspace vapor of the organic compounds of interest, after purification by a freeze-pump-thaw cycle, was leaked into the vacuum through a Granville-Phillips (Granville-Phillips Co., Boulder, CO) leak valve to maintain an indicated pressure of $8.0 \times 10^{-7}$ torr.

The ITSIM programs, versions 5.0 or earlier, can be used for simulations of trapping devices with cylindrical geometries, like 3D ion traps. To simulate ion motion inside an RIT, a newer version of the program, version 6.0, has been developed. The name "Ion Trajectory Simulation" (ITSIM) will be used for this and future versions with the program to emphasize the extended capabilities of simulating ion trajectories in electric devices of arbitrary geometries. The mechanical models of the RIT, generated by Mechanical Desktop 4.0 (AutoDesk, 2004), were input into a finite element 3D field solver FEMLAB 3.0a (COMSOL, Inc., Burlington, MA) and the electric field was solved with a maximum mesh element size of $0.8 \mathrm{~mm}$. The solved electric field was exported and converted into a field array file, which was used by ITSIM 6.0 beta version for simulation of ion trajectories under various experimental conditions.

\section{Results and Discussion}

The analytical performance of the 6-electrode RIT was optimized by using traps of stretched geometry with the inner RF electrode distance shorter in the $y$ - than in the $x$-direction [15]. The use of a higher DC voltage on the $z$-electrodes was also found to help improve the 
resolution by pushing ions towards the center of the RIT in the axial (z) direction [15]. Variation of the $\mathrm{z}$-electrode voltage was found to have significant effects on the trapping efficiency and ion trapping capacity of the 6-electrode RITs, both with or without stretched geometries. As shown in Figure 2, mass spectra of perfluorotributylamine (PFTBA) were collected using unstretched and stretched geometries with z-electrode voltages at 25,0 , and $-10 \mathrm{~V}$, while all other experimental conditions, including the leaked PFTBA vapor pressure, buffer bas pressure, ionization time, and the low mass cutoff (LMCO, at $m / z 50$ ), were kept identical for each experiment. The spectra were recorded using $2 \mathrm{~ms}$ ionization at a constant LMCO, $10 \mathrm{~ms}$ cooling time, and using a constant RF scan rate of $16,665 \mathrm{Th} / \mathrm{s}$. The frequency and amplitude of the resonance ejection $\mathrm{AC}$ signal was adjusted to maximize the ion signal intensity for each geometry. When the z-electrode voltage was set to $0 \mathrm{~V}$, the z-direction DC trapping potential well depth was $0 \mathrm{~V}$; however, the ions could still be trapped in the RIT, as shown in Figure $2 \mathrm{c}$ and $\mathrm{d}$. This phenomenon is due to the pseudopotential well resulting from the unbalanced RF when a dual-phase RF with unequal amplitudes for each phase is applied between $x$ - and y-electrodes. This method has been applied to allow simultaneous trapping of positive and negative ions in the linear ion trap [21]. With a single phase RF applied only to the y-electrodes, the pseudopotential well depth was maximized for each experiment. However, an RIT with the stretched geometries has a higher trapping efficiency when using an identical LMCO (Figure 2d). Decreases of $70 \%$ (Figure 2c) and 100\% (Figure 2e) in intensity were observed for unstretched RITs when the z-electrode voltage was dropped from 25 to $0 \mathrm{~V}$ and to $-10 \mathrm{~V}$, respectively, while little decrease was detected in the total ion current for the stretched RIT in both cases (Figure 2d and f). A significant signal decrease was observed for the stretched RIT when the z-electrode voltage was decreased below $-20 \mathrm{~V}$. The observed difference in the trapping efficiency suggests that the 6-electrode RIT with stretched geometry has a greater pseudopotential well depth, which helps compensate for z-axis ejection due to the DC potential.

To better understand the ion trapping behavior for both 6-electrode RIT geometries, field calculations and ion trapping simulations were carried out to illustrate the variation of the pseudopotential along the $\mathrm{z}$-axis under various conditions. For both the unstretched and stretched geometries, the distance between the grounded z-electrode and the ends of the RF electrodes was varied from 2.0 to $50.0 \mathrm{~mm}$. The electric field for each RIT configuration was solved using FEMLAB 3.0a with unit voltage applied to the y-electrodes, leaving the $\mathrm{x}$ - and z-electrodes grounded. The pseudopotential at the central point of $x-y$ plane on two ends of $R F$ electrodes was subsequently calculated for the ions

\section{End electrode voltage}

\section{Un-stretched, 6 electrodes $x_{0}=5.0 \mathrm{~mm}, y_{0}=5.0 \mathrm{~mm}$}

\section{Stretched, 6 electrodes $\mathrm{x}_{0}=5.0 \mathrm{~mm}, \mathrm{y}_{0}=3.8 \mathrm{~mm}$}

$25 \mathrm{~V}$

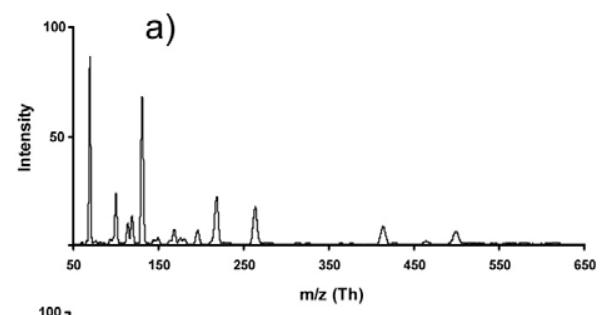

OV
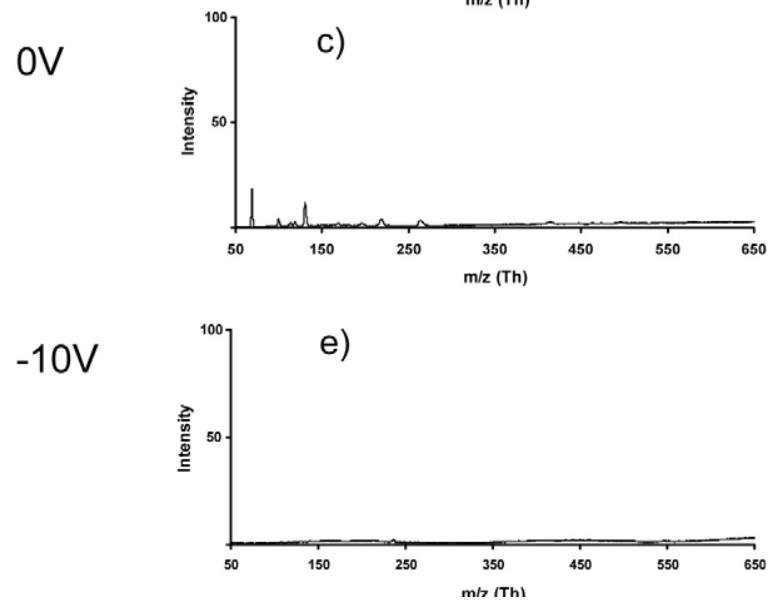
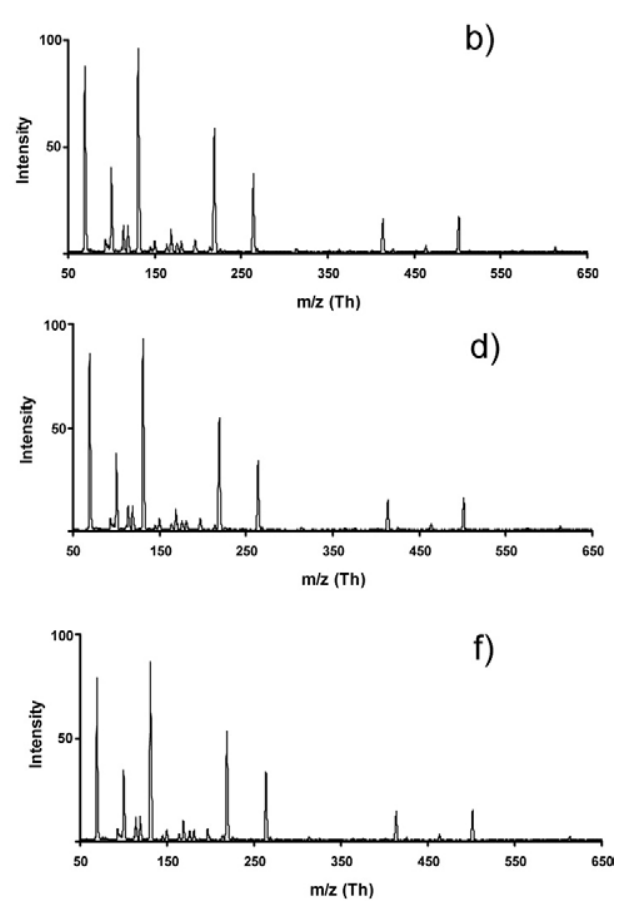

Figure 2. (a)-(f) Mass spectra of PFTBA collected using RITs of unstretched and stretched geometries with z-electrode voltages of 25,0 , and $-10 \mathrm{~V}$. 
under the condition $\mathrm{q}_{\mathrm{x}}<0.4$ using ITSIM 6.0 with the solved field and the following equation [28]:

$$
\Phi_{0}(x, y, z)=\frac{1}{4(m / e) \Omega^{2}} E_{0}^{2}(x, y, z)
$$

where $\Phi_{0}(x, y, z)$ is the static pseudopotential, $m / e$ is the mass-to-charge ratio of ion, $\Omega$ is the angular frequency of the applied $\mathrm{RF}$, and $E_{0}(x, y, z)$ is the amplitude of the electric field inside the device scaled up from that calculated using FEMLAB 3.0a. The pseudopotential was calculated for the ion $m / z 105$ with an RF of $200 \mathrm{~V}_{0-P}$ and 1.0 MHz. The pseudopotential for an RIT in $x-z$ plane can be plotted as shown in Figure 3a, and the pseudopotential wells along the z-axis were found for the all the RITs tested; the well depths varied significantly among the different configurations. The z-axis pseudopotential well is attributed to the RF fringing field caused by truncation, i.e., by the finite length of the $\mathrm{RF}$ electrodes, and it is usually smaller than that in the $x$ - and $y$-directions. The pseudopotential well depths along the $z$-axis were quantified as a percentage of the RF amplitude and plotted as a function of the distance between the z-electrodes and the end of RF electrodes for RITs with unstretched and stretched geometries,

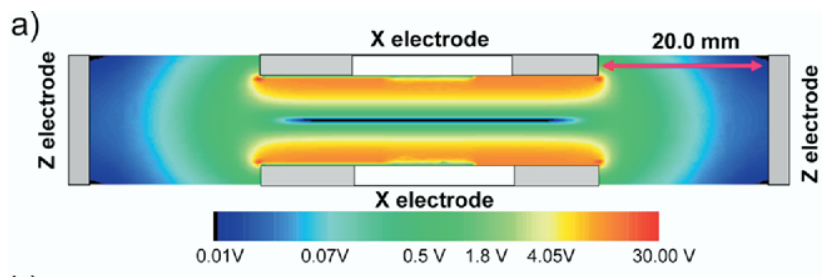

b)

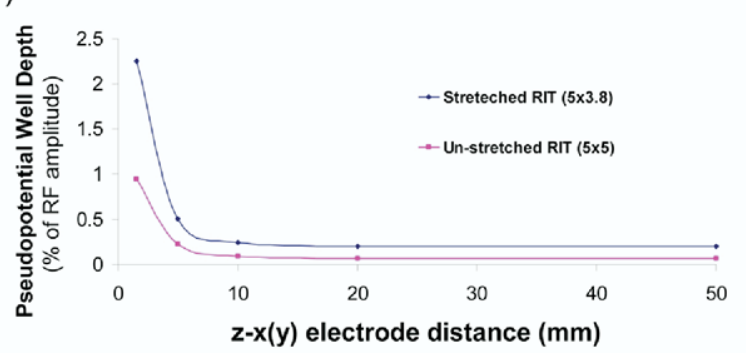

c)

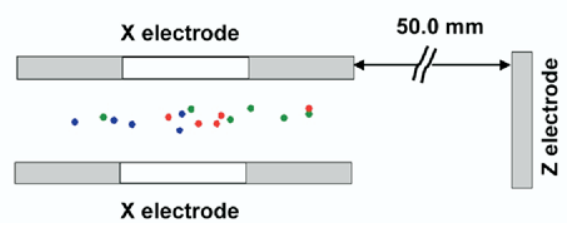

Figure 3. (a) Pseudopotential inside a stretched 6-electrode RIT with z-electrode $20.0 \mathrm{~mm}$ away from the end of RF electrodes, RF $200 \mathrm{~V}_{0-P}, 1.0 \mathrm{MHz}$; (b) pseudopotential well depth as a function of distance between the z- and RF electrodes, for a 6-electrode RIT with unstretched and stretched geometries; and (c) simulation of trapping ions $\mathrm{m} / \mathrm{z} 120,105$, and 77 inside a stretched RIT with z-electrode $50.0 \mathrm{~mm}$ away from the ends of RF electrodes, $10 \mathrm{~ms}$ after the generation of the ions from a $0.2 \mathrm{~mm}$ diameter spherical volume, $1.0 \times 10^{-4}$ torr He pressure, elastic ion-He collisions, and ion-ion columbic repulsion considered. respectively (Figure 3b). The well depth for each stretched RIT was found to be about twice that for the corresponding unstretched RIT when the same RF was applied. The most interesting observation from the calculated results is that the pseudopotential well depth decreases significantly with increasing spacing between the z-electrode and RF electrodes but approaches a constant value when the distance is larger than 15.0 $\mathrm{mm}$. The trapping of the ions in a stretched 6-electrode RIT with z-electrode gap of $50.0 \mathrm{~mm}$ was also simulated using ITSIM 6.0. The ions from acetophenone, $\mathrm{m} / \mathrm{z}$ 77, 105 , and 120, with abundances of 87, 100, and 30 for each $\mathrm{m} / \mathrm{z}$ value, were generated inside a spherical volume with a diameter of $0.2 \mathrm{~mm}$ at the center of RIT. An initial thermal energy of $\mathrm{kT} / 3(0.008 \mathrm{eV}$ at roomtemperature) along the $\mathrm{x}^{-}, \mathrm{y}^{-}$, and $\mathrm{z}$-directions, was given to each ion. An RF of $200 \mathrm{~V}_{0-p}$ and $1.0 \mathrm{MHz}$ was applied on the y-electrodes. A helium buffer gas pressure of $1.0 \times 10^{-4}$ torr, elastic ion-neutral collisions and coulombic repulsions among the ions (space charge condition) were used in the simulation. As shown in Figure 3c, the ions were spread out in the RIT after 10 $\mathrm{ms}$ due to the space charge effect and the collisions with the buffer gas molecules; however, 100\% trapping efficiency was achieved and no ions escaped in the zdirection (note that a maximum of only 16 ions can be shown as in Figure $3 c$ because of limitations in the graphical display during the simulation although all 217 ions were trapped in the simulation).

The z-electrodes of an RIT help to prevent penetration of external fields, serve as electric ground references, and contribute to the electric field distribution in the areas around the end of the RIT where the distance between the z-electrode and the RF electrodes is small in comparison with $\mathrm{x}_{0}$ and $\mathrm{y}_{0}$. However, in the cases where the z-electrodes are far from the RF electrodes, such as $50.0 \mathrm{~mm}$ or further for an RIT with an $\mathrm{x}_{0}$ of 5.0 $\mathrm{mm}$, it is reasonable to assume that the shape of the z-electrode should have little effect on the RIT performance. In practical terms, inside the vacuum manifold of an ion trap mass spectrometer, there almost always are electrically conducting objects within a distance of $50.0 \mathrm{~mm}$ from the mass analyzer that could serve as electric ground references for the RF. An RIT without z-electrodes should still have a pseudopotential well in the $\mathrm{z}$-direction when a single phase $\mathrm{RF}$, which is an extreme case of the unbalanced RF, is used.

To validate this hypothesis, RITs with only four RF electrodes were derived from the 6-electrode RITs by removing the z-electrodes. The 4-electrode RITs were installed into the vacuum manifold for tests, using a distance of $78.0 \mathrm{~mm}$ between each end of the RIT and the each side wall of the manifold (Figure 1). Mass spectra of PFTBA were recorded for unstretched and stretched geometries under the identical experimental conditions described above (Figure 4). With the same ionization time of $2 \mathrm{~ms}$ and optimized resonance ejection conditions, the ion intensity from the spectrum recorded with the stretched 4-electrode RIT is much 

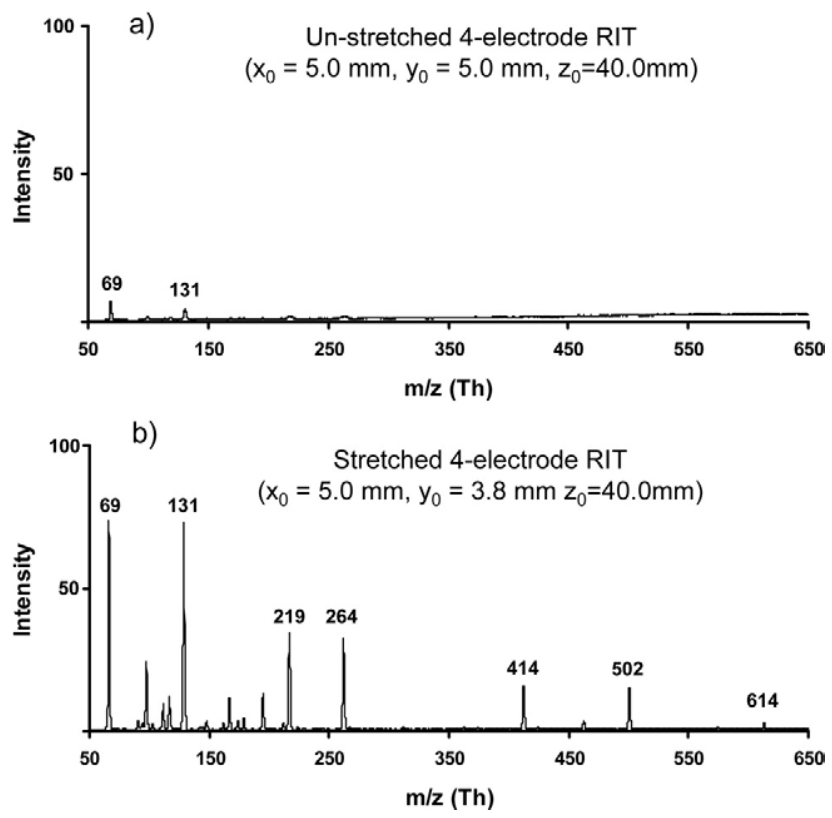

Figure 4. Mass spectra of PFTBA collected using 4-electrode RITs with (a) unstretched $\left(\mathrm{x}_{0}=5.0 \mathrm{~mm}, \mathrm{y}_{0}=5.0 \mathrm{~mm}\right)$ and $(\mathbf{b})$ stretched $\left(\mathrm{x}_{0}=5.0 \mathrm{~mm}, \mathrm{y}_{0}=3.8 \mathrm{~mm}\right)$ geometries, $\mathrm{z}_{0}=40.0 \mathrm{~mm}$.

higher, which indicates a higher trapping efficiency for this trap. This is also in agreement with the simulation result in which a deeper pseudopotential well was observed for RITs with stretched geometries.

A comparison of the overall trapping efficiency and ion trapping capacity was made amongst four RIT structures, namely, unstretched 4-electrode, stretched 4-electrode, unstretched 6-electrode, and stretched 6-electrode versions. In the tests using the 6-electrode RITs, a voltage of $25 \mathrm{~V}$ was applied to the z-electrodes. The experimental conditions were kept the same except that the ionization time was varied. Spectra of acetophenone were recorded as a function of the ionization time for each RIT. With a LMCO RF voltage of $120 \mathrm{~V}$, ions of $m / z 77,105$, and 120 were observed in each spectrum, for which the total ion intensity was calculated and plotted versus the corresponding ionization time (Figure 5). The stretched 4-electrode RIT was shown to have a trapping efficiency similar to the two 6-electrode RITs (unstretched and stretched), at ionization times shorter than $8 \mathrm{~ms}$. For the unstretched 4-electrode RIT, the z-axis pseudopotential is relatively shallow and the ions that gain kinetic energy from the driving RF or through the collision with the buffer gas could easily escape at the two ends of the RIT. At ionization time longer than $8 \mathrm{~ms}$, the stretched 4-electrode RIT reaches its maximum ion trapping capacity while the 6-electrode RITs are still responding to the increased ionization time. Much larger capacities are indicated for the RITs with an additional $25 \mathrm{~V} \mathrm{DC}$ trapping potential well along the $\mathrm{z}$-direction, which helps to prevent the escape of the ions caused by space charge effects [28].

The stability diagram has been used to characterize

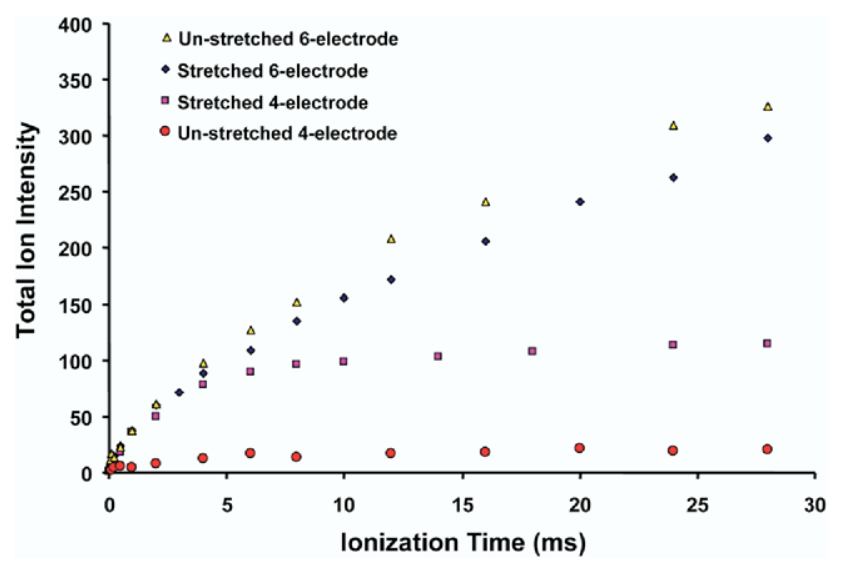

Figure 5. Comparison of sensitivity and trapping capacity among the unstretched and stretched RITs with or without the z-electrodes.

ion traps and to facilitate the design of control programs for tandem mass spectrometry [29]. The effect of elimination of the z-electrode on the RIT stability diagram was investigated by comparison between the stretched RITs with and without z-electrodes. The stability diagrams were mapped using the method previously reported [6, 15], and the fragment ion $m / z 105$ from acetophenone was used. The boundary of the stability diagram was found by varying the RF voltage and the DC offset applied on the RIT y-electrodes as shown in
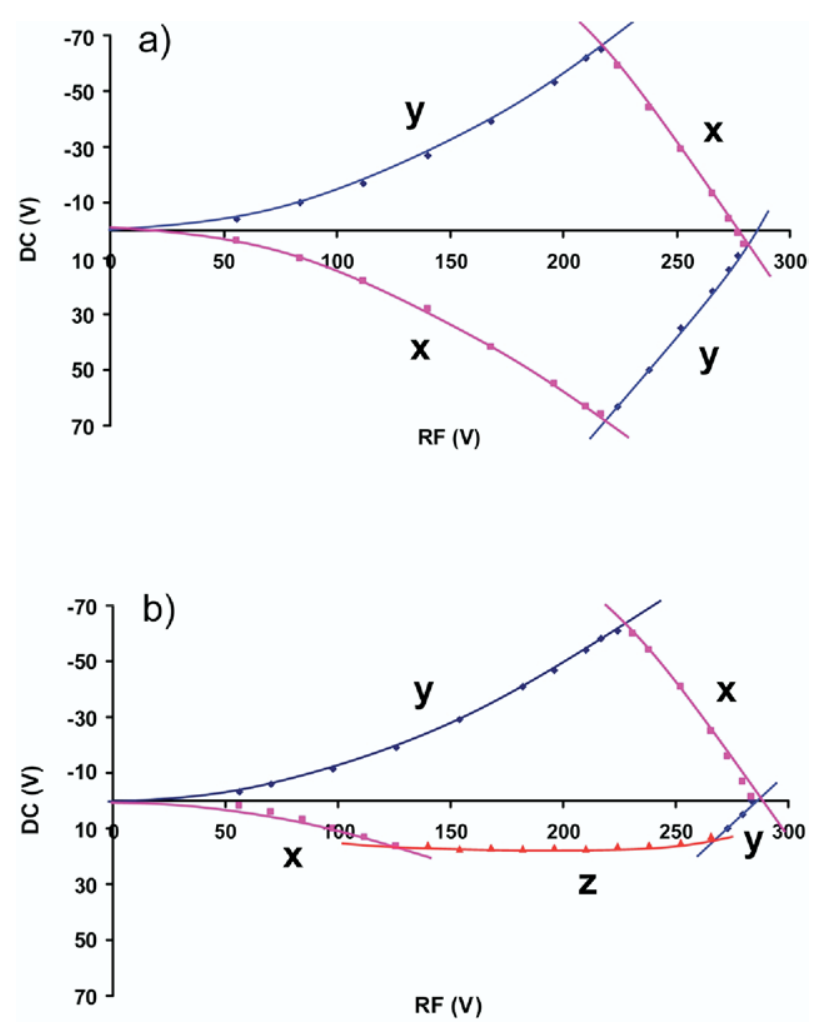

Figure 6. Stability diagrams mapped for (a) stretched 6-electrode RIT with 100 V DC applied on z-electrodes and (b) stretched 4-electrode RIT. 
Figure 6. The stretched 6-electrode RIT was shown to have a stability diagram similar to that of an unstretched RIT [15] except for a slight shift of the intercept of the $x$ - and y-boundary on the right side, which is similar to effects observed for 3D traps and is caused by the unequal dimensions in the $\mathrm{x}$ - and $\mathrm{y}$-directions. The top half of the stability diagram for the 4-electrode RIT is similar to that of the 6-electrode RIT, while the bottom half is flattened, for the ion $\mathrm{m} / \mathrm{z} 105$, at a DC offset voltage of $16 \mathrm{~V}$. When the RF offset DC voltage is increased, the center voltage of the RIT is also increased and an ejecting DC potential along the $\mathrm{z}$ axis is formed for positive ions. When the DC voltage is high enough to overcome the pseudopotential well generated in the $z$ direction by the $R F$, positive ions will become unstable in the $\mathrm{z}$ direction. As a result, the stability boundary in $z$-direction can be mapped as a function of the amplitudes of the RF and its offset DC, which is not the case for 6-electrode RITs.

The capability of performing the tandem mass spectrometry in a single device is a unique feature of the ion trap mass analyzer. The $\mathrm{MS}^{\mathrm{n}}$ capability of the 6-electrode RIT has been demonstrated [15] and fully characterized [16]. Although the 4-electrode RIT has been shown to have comparable MS capabilities using internal EI, it remained to be shown whether the MS ${ }^{\mathrm{n}}$ capabilities would be effectively retained when the $\mathrm{z}$-electrodes were removed. The isolation of the ions inside a 4-electrode RIT via RF/DC isolation proved to be applicable during the process of mapping the stability diagram. Additional experiments were carried out using notched SWIFT for precursor ion isolation and $\mathrm{AC}$ excitation of the selected ions to cause CID. Two stage MS/MS experiments were performed using a stretched 4-electrode RIT with the molecular ion $\mathrm{m} / \mathrm{z} 120$ of acetophenone as the starting precursor ion. A notched SWIFT was used to isolate the precursor ions at $q_{x}=0.64$ and a $100 \%$ isolation efficiency was achieved with a isolation window of $5 \mathrm{~m} / \mathrm{z}$. A resonant AC of 171 $\mathrm{kHz}$ frequency and $440 \mathrm{mV}_{0-p}$ amplitude was used to excite $\mathrm{m} / \mathrm{z} 120$ at $\mathrm{q}_{\mathrm{x}}=0.28$ for $30 \mathrm{~ms}$ and fragment ions $\mathrm{m} / \mathrm{z} 105$ were observed with a ca. 70\% CID efficiency. (Figure $7 \mathrm{a}, \mathrm{b}$, and $\mathrm{c}$ ) The fragment ion $\mathrm{m} / \mathrm{z} 105$ was further isolated using the same notched SWIFT and was excited at $\mathrm{q}_{\mathrm{x}}=0.28$ with using a second AC signal of $224 \mathrm{kHz}$ and $800 \mathrm{mV}_{0-p}$. Fragmentation of $\mathrm{m} / \mathrm{z} 105$ occurred and product ions of $\mathrm{m} / \mathrm{z} 77$ were observed (Figure $7 \mathrm{~d}$ and e). These CID conditions were similar to those used for the characterization of the CID capability of the 6-electrode RIT [15], and similar isolation and fragmentation efficiencies were observed with or without the z-electrodes. During the isolation and excitation of the ions, the collisions between the ions and buffer gas molecules could increase the ion momentum in the $z$-direction and cause the escape of the ions from the ends of RIT; however, the RF pseudopotential well seems to be deep enough to constrain the ions inside the RIT, according to the results from the experiments.
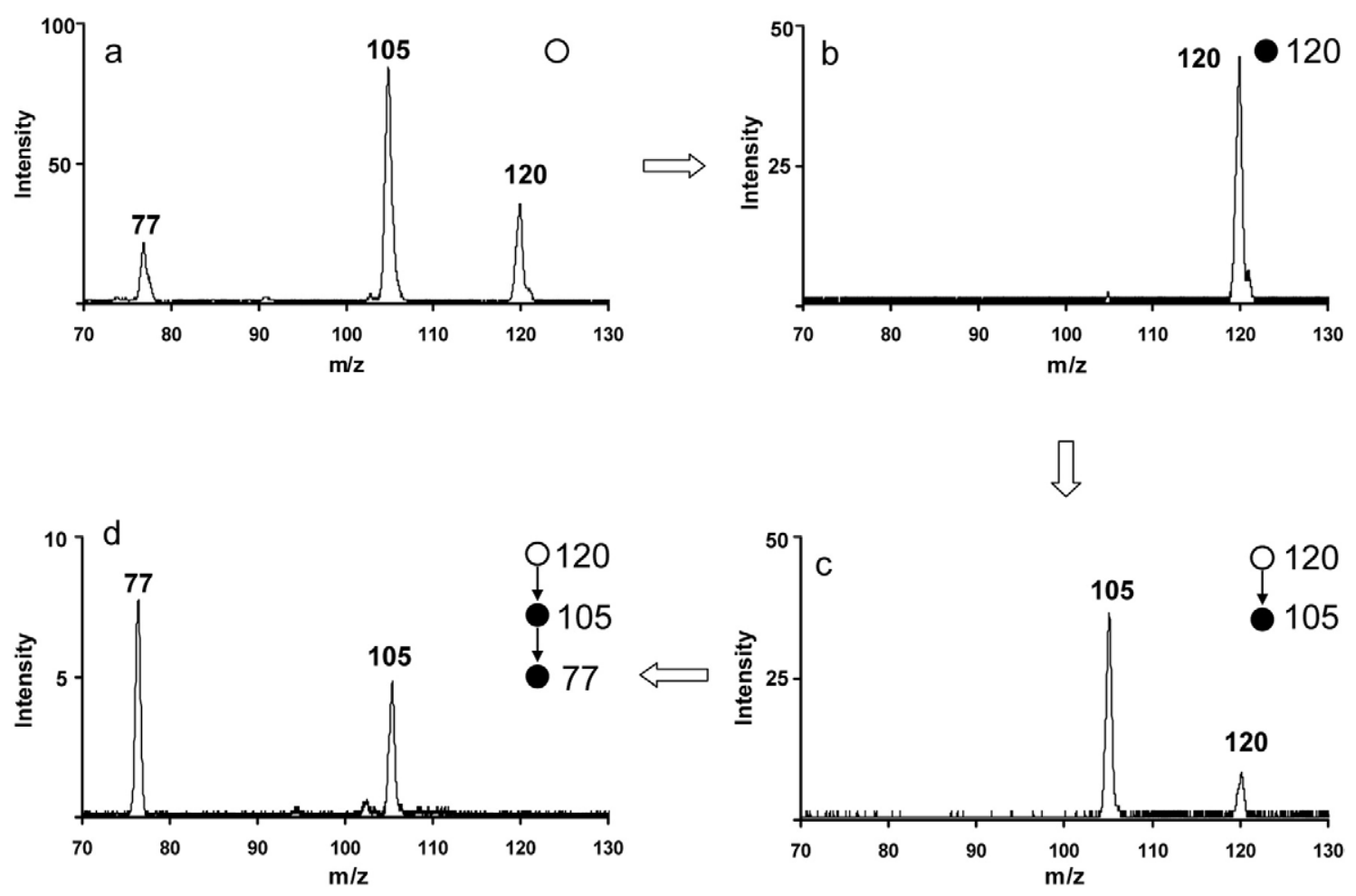

Figure 7. $\mathrm{MS}^{3}$ spectra of acetophenone collected using stretched 4-electrode RIT. (a) Spectrum of acetophenone; (b) molecular ion $\mathrm{m} / \mathrm{z} 120$ isolated using SWIFT notched at $\mathrm{q}_{\mathrm{x}}=0.64$; (c) product ion spectrum with excitation at $171 \mathrm{kHz}$ and $440 \mathrm{mV}_{0-p}$; and (d) sequential product ion spectrum of isolated $\mathrm{m} / \mathrm{z} 105$ with excitation at $224 \mathrm{kHz}, 800 \mathrm{mV}_{0-p}$. 
The RF pseudopotential well along the axis of the 4-electrode RIT has been demonstrated to be effective in trapping ions generated inside the RIT via EI and retaining them for MS and $\mathrm{MS}^{\mathrm{n}}$ analysis; however, the use of a pure pseudopotential well instead of a DC potential well along the z-axis for the linear ion traps is expected to cause a decrease in the trapping efficiency for ions injected externally in the z-direction due to the stronger effect of the alternating RF field along the $\mathrm{z}$-axis. The performance of the stretched RIT without $\mathrm{z}$-electrodes was tested in external ion injection and comparisons were made with the stretched 6-electrode RIT. The instrumentation is shown in Figure 8a with a modified Finnigan GCQ EI/CI source being used to provide ions. The acetophenone molecules were ionized by $70 \mathrm{eV}$ EI and the ions were delivered to the RIT using a three-lens system. The exit of the third lens is 2.0 $\mathrm{mm}$ away from the end of the RIT and a voltage of $-18.4 \mathrm{~V}$ was applied to it. Instead of a 4-electrode configuration for the tests with internal EI, a 5-electrode RIT is actually tested here with the third lens of the external ions source acting as a z-electrode. The RIT was floated at $-18 \mathrm{~V}$. A spectrum of acetophenone was acquired with an ionization time of $100 \mathrm{~ms}$ at an electron emission current of $1.5 \mu \mathrm{A}$ from the filament (Figure $8 b$ ). Spectra with similar signal-to-noise ratios were collected for the stretched 6-electrode RIT at a shorter ionization time of $20 \mathrm{~ms}$ with the help of DC potential well of just $0.2 \mathrm{~V}$ in $\mathrm{z}$ direction, which indicates a five times improvement in comparison with the 4-electrode RIT. The trapping efficiency for the exter-

a)

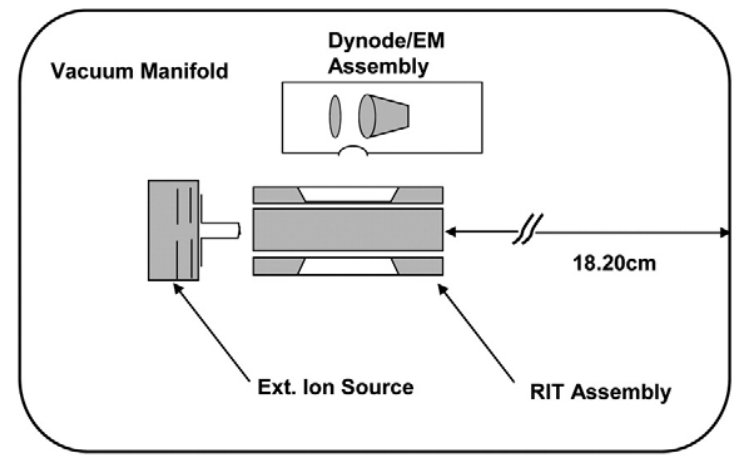

b)

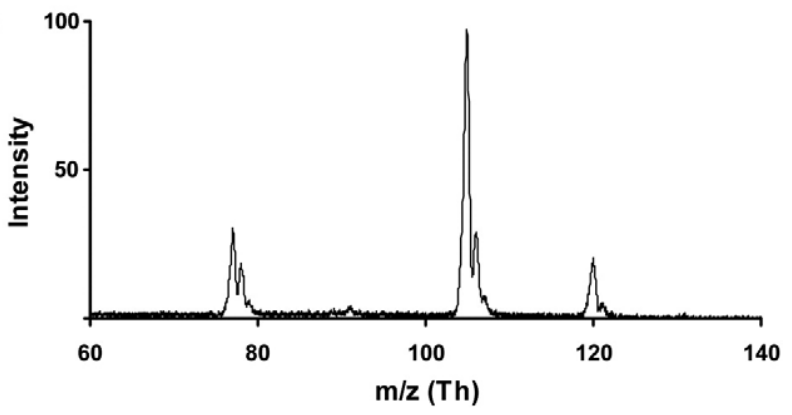

Figure 8. (a) Experimental setup for testing the 4-electrode RIT with external ion source and (b) mass spectrum of acetophenone collected using stretched 4-electrode RIT with ions injected axially into the RIT. nally injected ions in the traps using pseudopotential wells is estimated to be much lower than those using DC potential wells, typical values being $5 \%$ for $3 \mathrm{D}$ trap [30] versus up to $100 \%$ for linear ion trap [31]. A difference of a factor of five in the overall efficiency was observed between the 4-electrode and 6-electrode RIT in this experiment. The efficiency for the 4-electrode RIT, which was higher than expected, could have been improved by the ease of introducing the ions into the RIT given the open configuration without an additional electrode between the ion source and the RIT.

\section{Conclusions}

The 4-electrode RIT represents an additional simplification to the rectilinear ion trap geometry. It functions as a mass analyzer with adequate performance, i.e., simultaneous trapping of ions in a mass range up to $650 \mathrm{Th}$ with unit mass resolution, tandem mass spectrometry capabilities, and the ability to analyze externally injected ions. Three-dimensional ion trapping was achieved through a combination of radial trapping by the main RF and axial trapping by the RF fringe field axial components, which establish a pseudopotential barrier at each end of the four electrodes.

The use of the pseudopotential well in this device makes it a good candidate linear trap for instruments where ions with both positive and negative charges need to be trapped simultaneously. The simple structure of the 4-electrode linear ion trap makes it particularly significant in the development of miniaturized instruments. The trapping capacity loss accompanying decreases in the $x$ - and y-dimensions, to allow the use of lower RF voltages, can be compensated at least in part by increased trap length. The large opening in the z-direction allows a much higher injecting ion or electron current. It represents a vital step toward fabrication of massive arrays of miniaturized ion trap mass analyzer $[9,32,33]$, since this highly simplified device can be produced with considerably greater ease using metal coated glass tubes, which will largely reduce manufacturing costs and bring closer the time of the "throw away" mass analyzer.

\section{Acknowledgments}

The authors acknowledge funding from the Office of Naval Research (grant no. N00014-02-1-0834) and NAVSEA/NSWC (grant no. W912HZ-04-2-0001).

\section{References}

1. March, R. E.; Todd, J. F. J. Quadrupole Ion Trap Mass Spectrometry, 2nd ed.; John Wiley and Sons, Inc., Hoboken, NJ, 2005, pp 1-33.

2. Paul, W.; Steinwedel, H. A New Mass Spectrometer without a Magnetic Field. Z. Naturforsch., 1953, 8a, 448.

3. Stafford, G. C.; Kelley, P. E.; Syka, J. E. P.; Reynolds, W. E.; Todd, J. F. J. Recent Improvements in and Analytical Applications of Advanced Ion Trap Technology. Int. J. Mass Spectrom. Ion Processes 1984, 60, 85-98.

4. Syka, J. E. P. In Practical Aspects of Ion Trap Mass Spectrometry, Vol. I.; March, R. E.; Todd, J. F. J., Eds.; CRC Press: Boca Raton, FL, 1995; p 169. 
5. Franzen, J. The Nonlinear Ion Trap. Part 4. Mass Selective Instability Scan with Multipole Superposition. Int. J. Mass Spectrom. Ion Processes 1993, 125, 165-170.

6. Wells, J. M.; Badman, E. R.; Cooks, R. G. A Quadrupole Ion Trap with Cylindrical Geometry Operated in the Mass-Selective Instability Mode. Anal. Chem. 1998, 70, 438-444.

7. Wu, G.; Cooks, R. G.; Ouyang, Z. Geometry Optimization for the Cylindrical Ion Trap: Field Calculations, Simulations, and Experiments. Int. J. Mass Spectrom. 2005, 241, 119-132.

8. Kornienko, O.; Reilly, P. T. A.; Whitten, W. B.; Ramsey, J. M. Micro Ion Trap Mass Spectrometry. Rapid Commun. Mass Spectrom. 1999, 13, 50-53.

9. Blain, M. G.; Riter, L. S.; Cruz, D.; Austin, D. E.; Wu, G.; Plass, W. R.; Cooks, R. G. Towards the Hand-Held Mass Spectrometer: Design Considerations, Simulation, and Fabrication of Micrometer-Scaled Cylindrical Ion Traps. Int. J. Mass Spectrom. 2004, 236, 91-104.

10. March, R. E.; Todd, J. F. J., Eds.; Practical Aspects of Ion Trap Mass Spectrometry Vol. I: Fundamentals of Ion Trap Mass Spectrometry; CRC Press: Boca Raton, FL, 1995 pp 25-167.

11. Kocher, F.; Favre, A.; Gonnet, F.; Tabet, J.-C. Study of Ghost Peaks Resulting from Space Charge and Nonlinear Fields in an Ion Trap Mass Spectrometer. J. Mass Spectrom. 1998, 33, 921-935.

12. Schwartz, J. C. Proceedings of the 9th Sanibel Conference on Mass Spectrometry; Sanibel Island, FL, January 1997.

13. Schwartz, J. C.; Senko, M. W.; Syka, J. E. P. A Two-Dimensional Quadrupole Ion Trap Mass Spectrometer. J. Am. Soc. Mass Spectrom. 2002, 13, 659-669.

14. Hager, J. M. A New Linear Ion Trap Mass Spectrometer. Rapid Commun. Mass Spectrom. 2002, 16, 512-526.

15. Ouyang, Z.; Wu, G.; Song, Y.; Li, H.; Plass, W. R.; Cooks, R. G. Rectilinear Ion Trap: Concepts, Calculations, and Analytical Performance of a New Mass Analyzer. Anal. Chem. 2004, 76, 4595-4605.

16. Song, Q.; Kothari, S.; Senko, M. A.; Schwartz, J. C.; Amy, R. J. W.; Stafford, G. C.; Cooks, R. G.; Ouyang, Z. Rectilinear Ion Trap Mass Spectrometers with Atmospheric Pressure Interface and Electrospray Ionization Source. Anal. Chem. 2005, in press.

17. Zhang, C.; Chen, H.; Guymon, A. J.; Wu, G.; Cooks, R. G.; Ouyang, Z. Instrumentation and Methods for Ion and Reaction Monitoring Using A Nonscanning Rectilinear Ion Trap. Int. J. Mass Spectrom. 2005, in press.

18. Tabert, A. M.; Goodwin, M. P.; Cooks, R. G. Co-occurrence of Boundary and Resonance Ejection in a Multiplexed Rectilinear Ion Trap Mass Spectrometer. J. Am. Soc. Mass Spectrom. 2006, 17, 56-59.

19. Douglas, D. J.; Frank, A. J.; Mao, D. Linear Ion Traps in Mass Spectrometry. Mass Spectrom. Rev. 2005, 24, 1-29.

20. Xia, Y.; Liang, X.; McLuckey, S. A. Sonic Spray as a Dual Polarity Ion Source for Ion/Ion Reactions. Anal. Chem. 2005, 77, 3683-3689.
21. Xia, Y.; Wu, J.; McLuckey, S. A.; Londry, F. A.; Hager, J. W. Mutual Storage Mode Ion/Ion Reactions in a Hybrid Linear Ion Trap. J. Am. Soc. Mass Spectrom. 2005, 16, 71-81.

22. Zubarev, R. A.; Kelleher, N. L.; McLafferty, F. W. Electron Capture Dissociation of Multiply Charged Protein Cation. A Nonergodic Process. J. Am. Chem. Soc. 1998, 120, 3265-3266.

23. Pitteri, S. J.; Chrisman, S. A.; McLuckey, S. A. Electron Transfer Ion/Ion Reactions of Doubly Protonated Peptides: The Effect of Elevated Bath Gas Temperature. Anal. Chem. 2005, 77, 5662-5669.

24. Syka, J. E. P.; Coon, J. J.; Schroeder, M. J.; Shabanowitz, J.; Hunt, D. F. Peptide and Protein Sequence Analysis by Electron Transfer Dissociation Mass Spectrometry. Proc. Natl. Acad. Sci. U.S.A. 2004, 101, 95289533.

25. Louris, J. N.; Cooks, R. G.; Syka, J. E. P.; Kelley, P. E.; Stafford, G. C.; Todd, J. F. J. Instrumentation, Applications, and Energy Deposition in Quadrupole Ion-Trap Tandem Mass Spectrometry. Anal. Chem. 1987, 59, 1677-1685.

26. Guan, S. H.; Marshall, A. G. Stored Wave-Form Inverse FourierTransform Axial Excitation/Ejection for Quadrupole Ion Trap Mass Spectrometry. Anal. Chem. 1993, 65, 1288-1294.

27. Bui, H. A.; Cooks, R. G. Windows Version of the Ion Trap Simulation Program ITSIM: A Powerful Heuristic and Predictive Tool In Ion Trap Mass Spectrometry. J. Mass Spectrom. 1998, 33, 297-304.

28. Dehmelt, H. G. Radiofrequency Spectroscopy of Stored Ions I: Storage. Adv. Atom. Mol. Phys. 1967, 3, 53-72.

29. Todd, J. F. J.; Waldren, R. M.; Mather, R. E.; Lawson, G. On the Relative Efficiencies of Confinement of $\mathrm{Ar}^{+}$and $\mathrm{Ar}^{2+}$ Ions in a Quadrupole Ion Storage Trap (QUISTOR). Int. J. Mass Spectrom. Ion Phys. 1978, 28, 141-151.

30. Quarmby, S. T.; Yost, R. A. Fundamental Studies of Ion Injection and Trapping of Electrosprayed Ions on a Quadrupole Ion Trap. Int. J. Mass Spectrom. 1999, 190/191, 81-102.

31. Dolnikowski, G. G.; Kristo, M. J.; Enke, C. G.; Watson, J. T. Ion-Trapping Technique for Ion/Molecule Reaction Studies in the Center Quadrupole of a Triple Quadrupole Mass Spectrometer. Int. J. Mass Spectrom. Ion Processes 1988, 82, 1-15.

32. Misharin, A. S.; Laughlin, B. C.; Vilkov, A.; Takats, Z.; Ouyang, Z.; Cooks, R. G. High-Throughput Mass Spectrometer Using Atmospheric Pressure Ionization and a Cylindrical Ion Trap Array. Anal. Chem. 2005, 77, 459-470.

33. Tabert, A. M.; Griep-Raming, J.; Guymon, A. J.; Cooks, R. G. HighThroughput Miniature Cylindrical Ion Trap Array Mass Spectrometer. Anal. Chem. 2003, 75, 5656-5664. 